\title{
Ultra-spectra communication system (USCS): a promising way to energy-efficient green communication networks
}

\author{
Bin Li , Zheng Zhou and Weixia Zou
}

\begin{abstract}
In this article, we investigate a promising transmission approach for green communication networks. By combining the appealing single-cycle modulation technique with traditional pulse modulation found on the underlay communication philosophy, the presented ultra-spectra communication system can improve the energy efficiency significantly. In transceiver, the emission signal contains two parts, the information-bearing baseband waveform with an extremely short duration and the information-synchronized carrier component. Alternatively, without carrier signaling the intermittent emission can be adopted to further reduce its operational power. By optimally allocating the power in different bands, a water-filling emission spectrum inspired by a novel conception of radius basis function neural-network is proposed for maximizing transmission energy efficiency in the presence of external interference. In receiver, the carrier-synchronized signal greatly alleviates the power-consumed timing acquisition, and provides extremely precise synchronization for information detection in intensive multipath environments. In addition, from a pattern classification perspective, a low-complexity noncoherent detection scheme is suggested to enhance receiving performance and minimize energy consumption. Performance analysis demonstrated that the whole transmission scheme can indeed be realized within an ultra-low-power framework, which shows great advantages to traditional strategies.
\end{abstract}

Keywords: energy efficient, ultra-spectra communication system, single-cycle modulation, ultra-wideband, carrier synchronization, water-filling transmission, noncoherent detection

\section{Introduction}

The prevailing global warming and climate change have been a growing worldwide concern, which is mainly contributed to the unfettered emission of greenhouse gases, i.e., $\mathrm{CO}_{2}$ [1]. Mobile vehicles are generally considered as one potential enabler to reduce greenhouse gases provided that appropriate measures are implemented. The mobile industry itself also contributes to $\mathrm{CO}_{2}$ emission through communication links, mobile equipments, etc. Some analysts have reported that information and communication technology (ICT) accounts for $2-2.5 \%$ of all harmful emissions [2]. Meanwhile, as the large-scale distributed systems, such as smart grids, clouds, and P2P networks, gather and share more and

\footnotetext{
* Correspondence: stonebupt@gmail.com

Key Laboratory of Universal Wireless Communications, Ministry of Education (MOE); Beijing University of Posts and Telecommunications (BUPT), Beijing,
} China

more computing nodes and storage resources, the energy consumption keeps exponentially increasing, which has become significantly responsible for greenhouse gases emission. To keep in line with the low-carbon economy/industry development, it is necessary to reduce the operation expenditure or energy consumption of communication networks, while maintaining the acceptable quality of service (QoS) [3]. Many efforts are nowadays put into hardware specific solution as well as protocol designing, aiming at making contributions to the currently advocated energy-efficient green communications.

As a qualified potential candidate for short-range and high-speed transmissions, ultra-wideband (UWB) technology has been attached extensive investigations in recent years $[4,5]$. Usually, UWB refers to communication systems with the spectral occupancy larger than $500 \mathrm{MHz}$, or the fractional bandwidth more than $20 \%$ 
[6]. The fractional bandwidth is defined as $W / f_{c}$, where $W=f_{H^{-}} f_{L}$ denotes the $-10 \mathrm{~dB}$ bandwidth and the center frequency is $f_{c}=\left(f_{H}+f_{L}\right) / 2 \times\left(f_{H}-f_{L}\right)$. To generate such signals, data symbols are usually carried by a set of baseband short pulses with the duration less than $1 \mathrm{~ns}$ [7]. According to the classical Shannon channel capacity theory and by virtue of its huge transmission bandwidth, the data rate of UWB system may easily surpass $1 \mathrm{Gbps}$ within $10 \mathrm{~m}$, which is much attractive for real-time multimedia services [8]. What's more, the remarkable lowduty-cycle (LDC) operation of UWB can be promised, in which information bits are carried by short-duration pulses and emitted through antenna only in very limited spans of time, as suggested by the popular schemes such as pulse position modulation (PPM) [5,6]. Attributed to this unique intermittent emission manner, which is idle most of the time and only active for a transitory period, UWB may conduct data transmissions at an extremely low power $(<-41.3 \mathrm{dBm} / \mathrm{MHz})$ by elegantly addressing spectrum efficiency and energy efficiency through an underlay communication philosophy [5,7]. In consideration of its high potential in various short-range applications in large-scale distributed networks, such as environment pollution sensing, intelligent traffic guiding and remote health monitoring [9], UWB based lowradiation techniques are of great promise to future energy-efficient green communications.

Due to its enormous emission bandwidth, nevertheless, UWB still faces great challenges in system designs and implementations. First, the typical UWB propagations are highly dispersive into hundreds of resolvable multipath trajectories $[10,11]$. As the detection performance is noticeably sensitive to timing deviation, the analog to digital convertor (ADC) with a sampling frequency of $20 \mathrm{GHz}$ is indispensable in timing acquisition [12], which is hence essentially contrary to the spirit of low-power and low-complexity receiver structure. Second, given the strictly regulated spectra limits remaining plat in most frequency band [13], the nonflatly distributed interference spectra call for judicious baseband waveforms designing to enhance the transmission energy efficiency. Lastly, for traditional UWB receiver architecture, a large number of Rake fingers should be integrated, which arouses hardware implementation challenges and simultaneously consumes considerable power [14]. Also, the complicated channel impulse response (CIR) estimation needs much energy. Even for the transmitted-reference (T-R) scheme, the energy efficiency (e.g., typically about $50 \%$ ) may be greatly satisfied as a compromise of simple realizations [15]. Apparently, these practical difficulties either complicate system design or greatly deteriorate the appealing low-power characteristics, making UWB based green communications not available.
Motivated by the considerations above, in this research we suggest an attractive energy efficient communication scheme. The systematic transmission strategies, including the modulation scheme and optimal waveform shaper design in transmitter and the lowcomplexity low-power synchronization as well as detection technique in receiver, are thoroughly addressed under realistic operational scenarios, i.e., in the presence of external interference and intensive multipath. During this new transmission approach, the single-cycle modulation (SCM) technique is organically combined with classical pulse modulation [16]. The derived emission technique can be regarded as a carrier-synchronized UWB [17]. As the emission signal exhibits an extremely broad continuous spectrum (i.e., an idea of UWB) accompanying an ultra-narrow single-carrier spectra in the center (i.e., a conception of ultra-narrow band according to power bandwidth definition), from a unified perspective it can be referred to as ultra-spectra communication system (USCS). The underlay spectrum conveys information in an ultra-low-power fashion, while the carrier signaling may facilitate precise synchronization in detection. The main contributions of our investigation on USCS can be summarized into the following aspects:

(1) In USCS transceiver, we firstly suggest a versatile waveform shaper which is inspired by the novel conception of radius basis function neural network (RBFNN). Starting from the Shannon channel capacity theory, a water-filling (WF) emission spectrum is suggested and then realized by the appealing RBFNN. Relying on the function approximation ability and fast convergence of RBFNN, the transmission energy efficiency can be maximized given arbitrary external interferences in working band. By integrating intermittent emission (i.e., UWBIR) and carrier synchronized mode, furthermore, the suggested reconfigurable hybrid USCS transceiver may make ultra-low-power transmission into practice.

(2) After propagated from the intensive multipath channel, in received USCS signal the strong carrier component can be conveniently extracted by a phase locked loop (PLL), which provides accurate synchronization/ timing to subsequent signal detection, leading to a much low ADC sampling rate accompanying low-power structure. Meanwhile, compared with existing UWB system, the precise synchronization may also significantly alleviate the requirements on signal-to-noise ratio (SNR) in receiver, and hence can minimize emission power in transmitter given the expected QoS and operation condition. Beside, by incorporated with wireless charging techniques [18], the strong energy in synchronized carrier signaling may be used for remote powering, which may provide a feasible energy maintenance solution to super large-scale sensor networks. 
(3) In USCS receiver, remarkably different from the power-consumed coherence receivers (e.g. Rake receiver or T-R structure), from a pattern classification point of view we developed a low-complexity noncoherent receiver architecture based on the fuzzy c-means clustering algorithm (FCM) [19]. This blind detection can considerably reduce energy consumption and also enhance receiving performance, which is hence extremely attractive for the use in low-complexity wireless sensors and body area networks.

The reminder of this article is outlined as follows. In Section 2, after a brief introduction to UWB technique, we depict the new systematic communication framework, i.e., USCS. The systematic strategies in transceiver are investigated in Section 3, including the emission schemes and optimal waveform design for energy-efficient transmissions. In receiver, the simple synchronization scheme is discussed, and then a low-complexity noncoherent detection is proposed to further reduce energy consumption. Subsequently, in Section 4 the performance of our USCS is thoroughly analyzed from an architecture aspect of energy efficiency. Finally, we conclude the whole work in Section 5.

\section{Ultra-spectra modulation}

Currently, many different physical technologies have been suggested for UWB systems, including the multiband orthogonal frequency division multiplexing (MBOFDM) based UWB [8], the continuous pulse UWB [20] and classical impulse UWB [5,8]. It is gradually recognized that, even though with the higher spectra efficiency and more preferable transmission speed, usually the complex signal processing for continuous modulations (e.g., power amplifier or up/down conversion) consuming considerable power may significantly degrade their energy efficiency [21], and also complicate the practical realization. The appealing low-power radiation property of impulse UWB is essentially attributed to the accommodation of a compromise between spectrum and energy efficiency. And in sharp contrast, the intermittent impulse transmission and the resulting LDC property, as well as feasibly simple noncoherent detection, may greatly reduce its energy consumption. In this section, by incorporating the LDC impulse radio (IR) technique with SCM, we present a promising emission technique for green communications, which may lay the foundation of energy efficient transmissions. Before further elaboration, it is necessary to briefly describe the underlay philosophy based UWB-IR system.

\subsection{UWB system}

Without the adoption of local oscillators or mixers, the typical UWB signal can be generated by driving an RF antenna with extremely short pulses, whose duration is on the order of a few nanoseconds to fractions of a nanosecond $[5,8]$. Accordingly, the modulated UWB signal is an impulse-like waveform. Therefore, UWB is often referred to as a short-pulse or IR system. In our analysis, we employ the classical time-hopping PPM (TH-PPM) [7], and the signal format is described by

$$
x(t)=\sqrt{E_{b} / N_{s}} \sum_{i=0}^{M-1} g\left(t-i T_{f}-c_{i} T_{b}-d_{\left\lfloor i / N_{s}\right\rfloor} \Delta t\right)
$$

where $E_{b}$ is the bit energy, $N_{s}$ is the number of pulses used to represent one bit, $T_{b}$ is the bit period of a single bit, $d(i)(i=0,1,2, \ldots, M-1)$ are the transmitted information data taking values of $\{0,1\}, T_{f}$ is the time period of a frame, $c_{i}$ is the time hopping code and $\Delta t$ is the bit separation time interval for one PPM symbol. Particularly, $T_{f}=T_{b}$ for $N_{s}=1 . g(t)$ is the adopted pulse shaper, which is usually the second derivative Gaussian pulse with a parameter $\delta[5,6]$ :

$$
g(t)=\left(1-4 \pi t^{2} / \delta^{2}\right) \times \exp \left(-2 \pi t^{2} / \delta^{2}\right)
$$

A typical second-derivative Gaussian pulse and the corresponding emission spectra of TH-PPM scheme have been plotted by Figure 1. From the illustration in Figure $1 \mathrm{~b}$, we may note that the emission spectrum is basically determined by the baseband waveform $g(t)$, while the information-bearing $\mathrm{TH}$ coding does not significantly alter the spectra shape [22].

\subsection{USCS}

Originally, the SCM technique is also derived for lowpower applications in which each information bit is exactly modulated within one single carrier period $[23,24]$. Unfortunately, for the currently existing SCM schemes, the front-end filter design still remains complicated and even confusing [25], which is supposed to removing the useless high-harmonics accompanying the underlay broad spectra, while also preserving the discontinuous points in time domain and hence retaining acceptable detection performance. Moreover, the energy efficiency of such a technique can not be validated by classical theoretical analysis [26]. To response the practical and theoretical challenges, we design a new modulation scheme by introducing the random pulse position keying technique into SCM [16].

As a consequence, the corresponding baseband modulated signal $s(t)$ can be written as:

$$
s(t)=A \sum_{n=-\infty}^{\infty} a_{n} g\left(t-n T_{f}-B_{n} T_{1}-T_{n}\right)+D
$$

The emission signals can be generated after a carrier modulation, such as phase shift keying (PSK), has been 


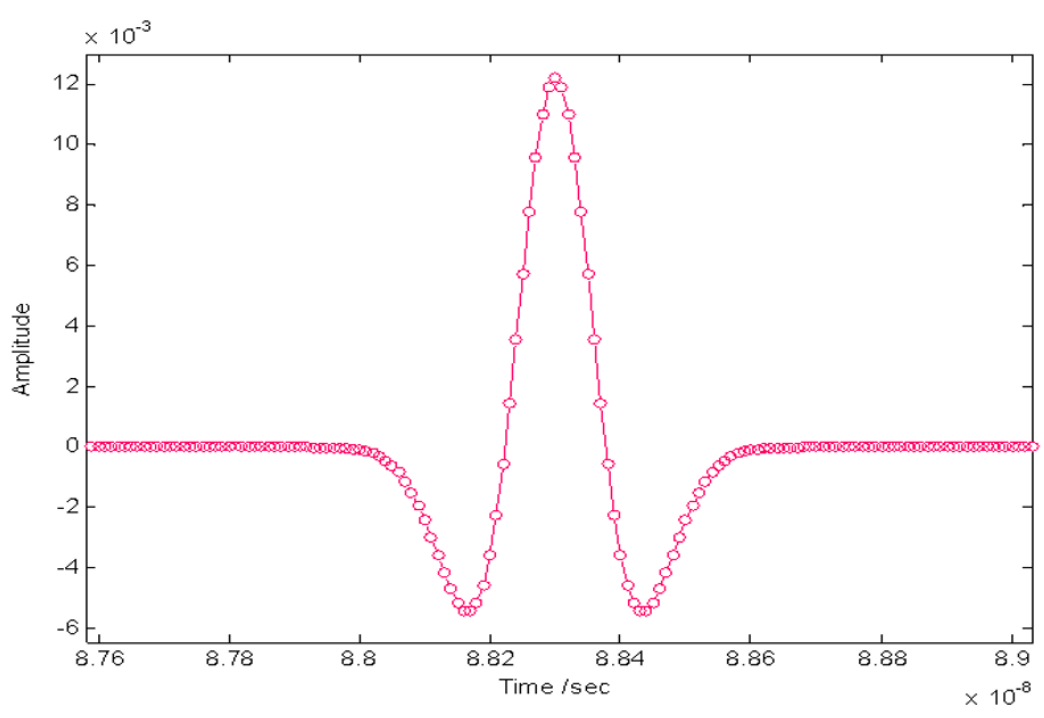

(a)

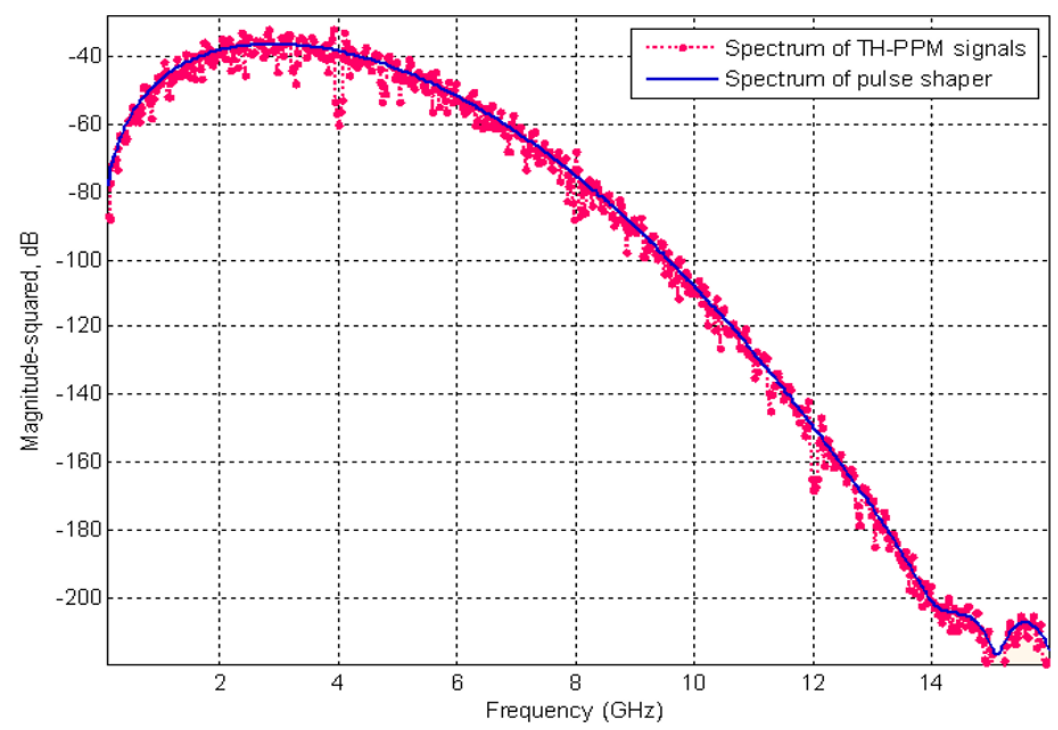

(b)

Figure 1 UWB-IR signal. (a) Waveform of pulse shaper; and (b) emission spectra of TH-PPM. Here, we set $\delta=3 \times 10^{-10} \mathrm{~s}$

performed on this baseband signal. It is worth noting that the main difference of (3) is the additional directcurrent (DC) component denoted by $D$, which corresponds to an ultra-narrow carrier spectrum in frequency domain. Therefore, such a system can be also viewed as a kind of carrier-synchronized UWB (CS-UWB) [17]. Based on SCM conception, it should be emphasized that the carrier period $T_{c}$ and the duration of $g(t)$ (i.e., $T_{g}$ ) should meet the specific constraint, i.e., $T_{g}=T_{\mathrm{c}}[16,23]$.

The schematic diagram of USCS is illustrated in Figure 2. First, an periodical impulse sequence $\Sigma_{n} q\left(t-n T_{f}\right)$ is generated. After this sequence passed a delayed modulator, the pulse position is accurately controlled by a prescribed user code $B_{n}$ and a random delay $T_{n}$. That is, the time delay of current impulse is measured by $B_{n} T_{1}$ $+T_{n}$ [16]. Then, the position modulated signal is fed into a pulse shaper, and multiplied by information bits $a_{n} \in\{-1,1\}$. The USCS baseband signal can be obtained after superimposing DC component. Finally, the signal is emitted to wireless channels after single carrier modulation has been performed on baseband signal, as shown by Figure 3a. It should be noted that, instead of simple superposition, the information-bearing pulse is exactly synchronized with the carrier signaling during one single period. That is, the information pulses happen to occupy the single carrier period, with their start and cut points 


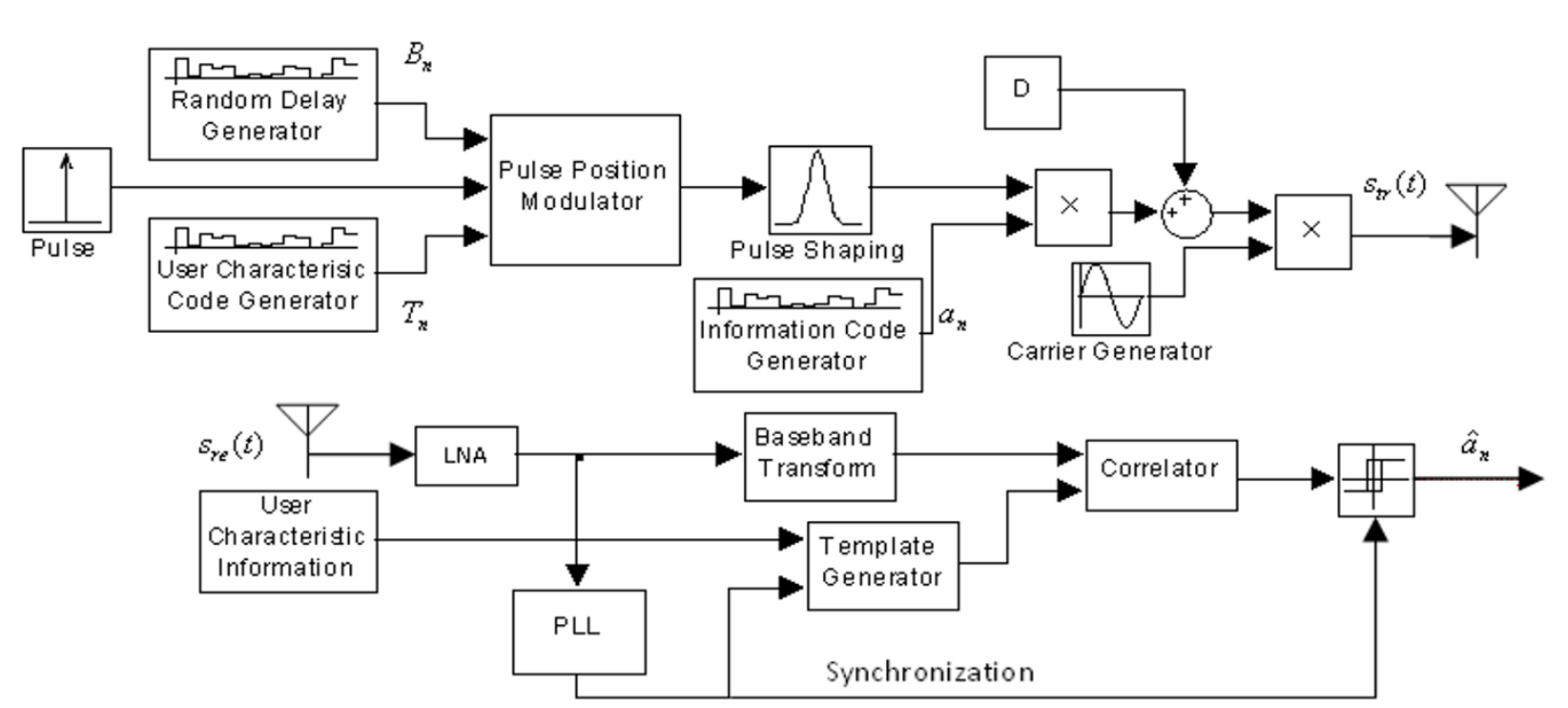

Figure 2 Schematic structures of USCS.

precisely aligned (or synchronized) with one carrier period.

For this special CS-UWB, the underlay continuous spectrum is much similar to that of Figure $1 \mathrm{~b}$ when adopting a same pulse shaper. While an obvious singlefrequency carrier component appears above the continuous spectrum, as is illustrated by Figure 3b, which is located at about $2.5 \mathrm{GHz}$. The amplitude of the singlecarrier is related to $D$ and the designed LDC value. For simplicity, in simulation we set $A=3$ and $D=1$ and the resulting LDC value is $30 \%$. The amplitude difference between the single-carrier spectra and continuous spectra is about $40 \mathrm{~dB}$. Notice that, a practical LDC of $1 \%$ may produce an even higher narrow carrier spectrum. According to the U.S. FCC recommend on bandwidth definition, there should be the total $99 \%$ signal power hold in band. Such a power reservation criterion is practically equivalent to the $-20 \mathrm{~dB}$ attenuation bandwidth, indicating that spectral attenuation from the peak power to the cutoff frequency point is no less than 20 $\mathrm{dB}$. In some other literatures, from a power bandwidth rather than information bandwidth [23,27], such a system can be referred to as ultra-narrow band system (UNB), whose $-40 \mathrm{~dB}$ attenuation bandwidth is only several Hertz [16]. On the other hand, based on the conception of SCM in which signal bandwidth (i.e., continuous spectrum) is roughly the quadruple of carrier frequency, that is, the fractional bandwidth is about $50 \%$ (i.e., larger than 20\%). Hence, it is actually a special UWB system from Figure 3b, from a relative bandwidth perspective [5]. This is the main reason, in this study, why we mentioned this transmission strategy as USCS.
The main philosophy behind USCS is to disperse a small portion of energy into a flat continuous-sideband spectrum, and simultaneously, to minimize bandwidth of the single-carrier spectra. Accordingly, the emission spectrum can be then divided into two separate parts, i. e., the narrow carrier spectra and the remaining sideband spectrum, which can be shuffled by resorting to a PLL device [28]. Following the Fourier transform, the useful information of USCS signal is involved in continuous spectrum, while the single-carrier component may greatly facilitates timing process.

\section{Transmissions by USCS}

\subsection{Emissions in transceiver}

\subsubsection{Intermittent emission or continuous emission?}

For conventional communication schemes, the information bearing waveform always occupies the whole symbol duration, as is for the classical binary PSK (BPSK) [28]. In contrast, in an intermittent impulse system the transceiver conducts emission only in a much small portion of symbol duration. And the resulting LDC only approaches $1 \%[5,29]$. As a consequence, the overall transceiver power consumption can be expected to keep below $1 \mathrm{~mW}$, while providing a data rate of $10 \mathrm{Mbps}$ with an acceptable QoS [30]. Although this incomparable energy-efficient transmission of UWB-IR, as indicated by most investigations $[11,12,14]$, the receiver synchronization process as well as sophisticated correlation-based detection is generally energy-consumed in the presence of highly dispersive propagations, which may significantly offset the advantages of UWB based green transmissions. 


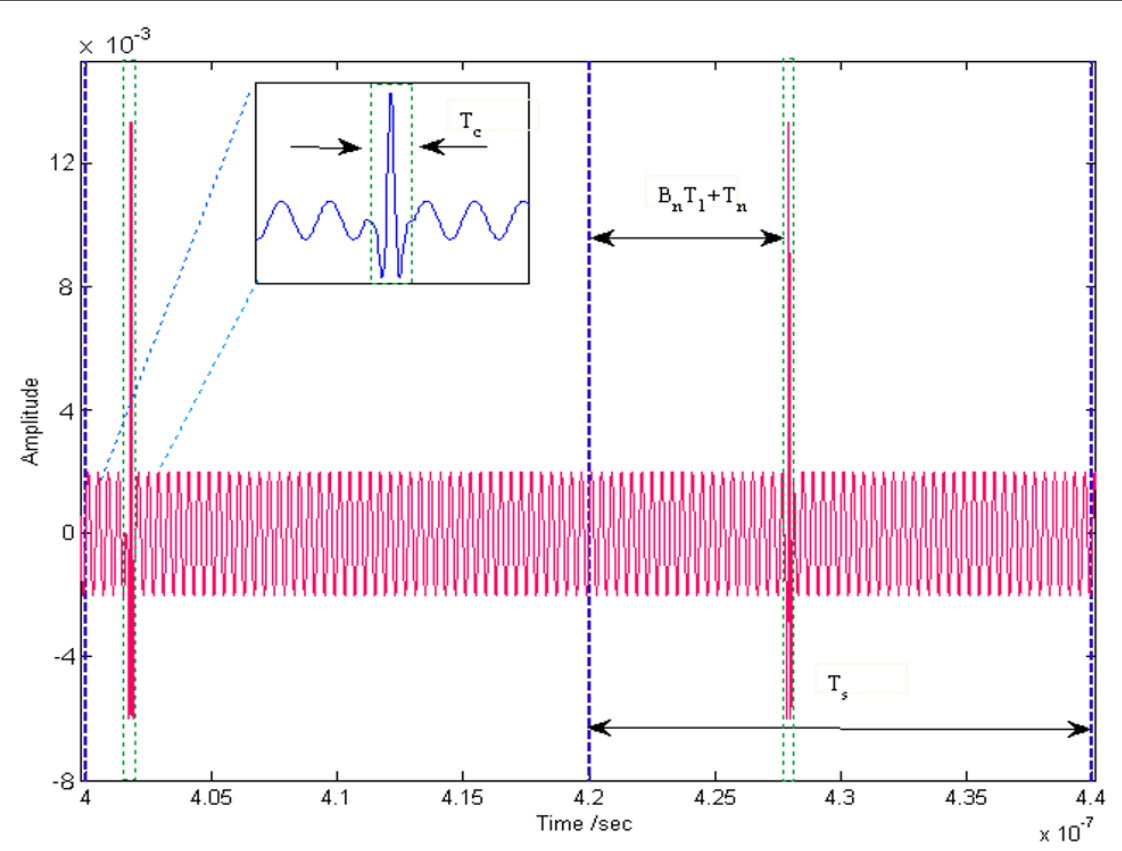

(a)

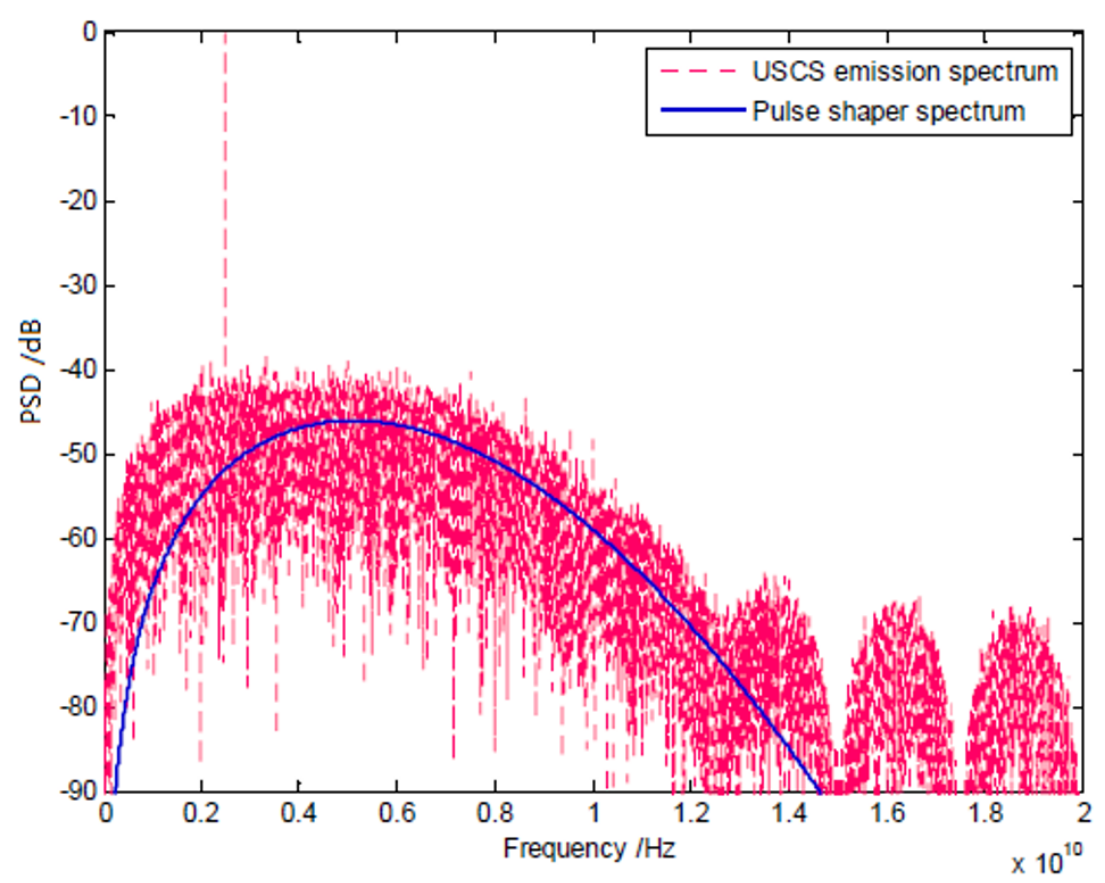

(b)

Figure 3 USCS signal. (a) Emission waveform of USCS; and (b) USCS emission spectra. Notice that a pulse shaper in (2) is used.

On the other hand, in USCS the signal is continuously emitted attributed to the carrier-synchronized waveform. The information is carried by a spreading continuous spectrum, and the synchronized-carrier reference can directly provide accurate timing for receivers. Furthermore, by employing the low-complexity noncoherent detection techniques, such a transmission scheme can be realized within a low-power framework. Nevertheless, 
it is noted that the carrier component only serves as the timing reference, and hence, a directly raised question is that is it necessary to use such an energy redundant synchronization signaling?

First, it has to be emphasized that precise synchronization is of immense significance to the detection of short-duration information waveform as in USCS. Specifically, a practical $1 \%$ timing error with respect to pulse duration may degrade the detection performance by 20 $\mathrm{dB}$ (or even much) for impulse UWB [5,29]. So, it is indeed of promise to integrate a precise synchronization reference in emission signal to reinforce receiver performance (see Section 3.2). A comprehensive energy efficiency analysis in this situation will also be found in Section 4.1. Moreover, other than facilitating accurate timing, this highly strong single-frequency component can be easily extracted from received signals by using a simple PLL, so that the recovered energy can be further collected to compensate energy consumption in receivers [18]. If we assume the emission power in intermittent mode is $0.5 \mathrm{~mW}$, then taking a LDC value of $1 \%$ for example, for continuous USCS the total carrier energy may reach $49.5 \mathrm{~mW}$. Assuming a conservative energy conversion ratio of $70 \%$, then the receiver can basically collect an additional energy of $3.1 \mathrm{~mW}$ from the information-bearing signals, when operating in a typically short-range scene with a propagation attenuation of $-10 \mathrm{~dB}[11,28]$. This accumulated energy may even entirely cover the energy consumption for a long detection period. Thus, such a carrier-synchronized signal can be fully explored to provide an attractive remote powering approach to large-scale wireless sensor networks (WSNs). Notice that, however, energy efficiency of this remote powering solution may be degraded in long-range applications.

Furthermore, a much more preferable way is to combine the above two transmission strategies together. For such a reconfigurable USCS transceiver, when the local clock deviation has exceeded the tolerable error threshold according to the synchronization drifting property, we may use the continuous transmission (i.e., the carrier-synchronized modulation) to correct timing deviations, and simultaneously, conduct energy reservation from received signals for the purpose of prolonging lifetime of low-cost battery-powered sensor nodes. Once the timing calibration has been completed, or sufficient energy has been accumulated, we may immediately switch to an intermittent mode. In such a hybrid case, the operational power of USCS receiver can be minimized, while ensuring a substantial transmission performance with the assistance of simple and accurate timing technique accompanying low-complexity noncoherent detection.

\subsubsection{Water-filling emission spectrum}

Motivated by the current trends of high-speed transmissions, the appealing techniques such as OFDM and direct-sequence spread spectrum are widely used in wireless services [31]. As a consequence, for USCS occupying $f_{B}=\left[\begin{array}{ll}0 & 12.5\end{array}\right] \mathrm{GHz}$, the external interference power spectrum $I(f)$ from other systems can be reasonably viewed as Gaussian noise. Assuming the in-band interference power is $\sigma^{2}$, we have

$$
\int_{f_{B}} I(f) d f=\sigma^{2}
$$

On the other hand, for the purpose of elegant spectral coexistence, the underlay spreading spectrum (SS) of USCS should also be strictly restricted by regulatory emission mask [13]. The total USCS power during authorized band is limited by $P$.

$$
\int_{f_{B}} G^{2}(f) d f \leq P, G^{2}(f) \leq M(f)
$$

where $M(f)$ denotes the emission mask regulated by radio management [13], and $P=\int M(f) d f$. According to the Shannon channel capacity theory [28], the capacity can be expressed into

$$
C_{\text {USCS }}=\max _{G(f)} \int_{f_{B}} \log [1+G(f) / I(f)] d f
$$

under spectral constraint:

$$
G^{2}(f) \leq M(f)
$$

Consequently, in order to maximize channel capacity of USCS and simultaneously improve transmission energy efficiency, the information-bearing waveform (i.e., $g(t))$ should be carefully designed. By resorting to the well known Lagrange multiplier technique [28], the optimal emission spectra can be easily derived [32]:

$$
G(f)=\sqrt{M(f)}-I(f)
$$

The above obtained emission spectrum is known as the water-filling (WF) spectrum. Given the total emission power $P$ and the nearby networks interference, the objective is to generate baseband pulse entirely matching the target spectrum $G(f)$. At the same time, the designed spectrum should keep below $M(f)$ for coexistence when $I(f)=0$.

In the sense of energy-efficient transmission, it is obvious that the high-order derivatives of Gaussian pulse may be not a good choice any more [32]. Relying on a novel conception of RBFNN, in this part, we 


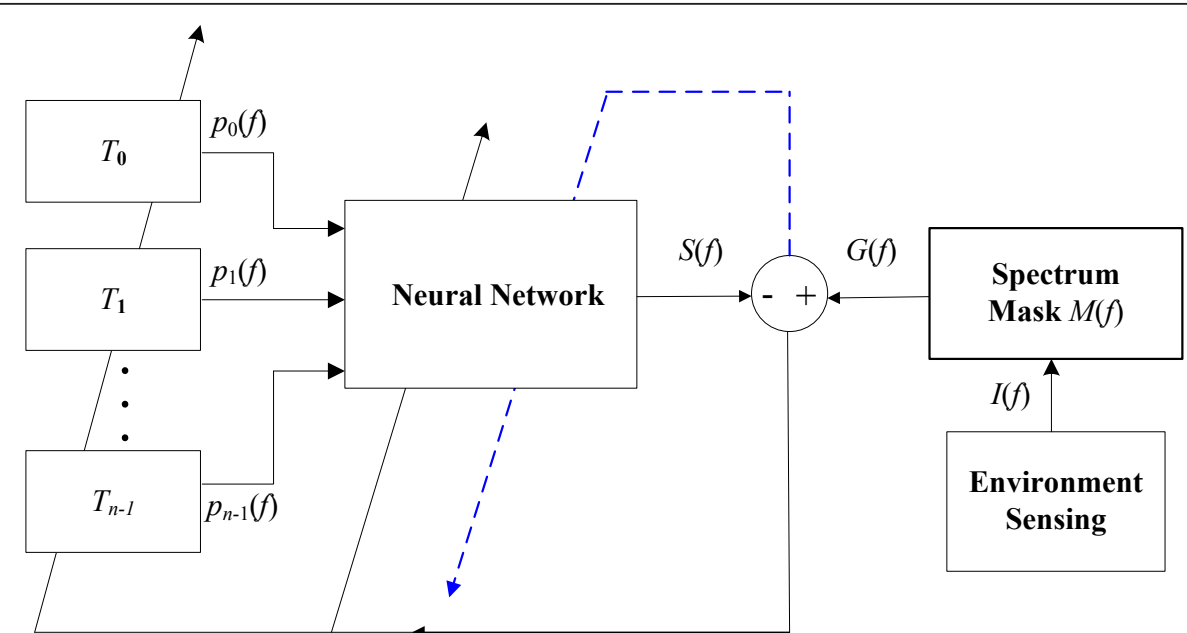

Figure 4 Implementation structure of RBFNN based waveform shaper.

suggest an optimal WF waveform design technique. Owing to the excellent ability of function interpretation and network adaptation of this generalized shaper [33], the designed signal is highly reconfigurable which may also entirely match the target spectral shape $G(f)$ after a short switching time.

The implementation architecture has been shown in Figure 4. Based on environment sensing results, the target output $\mathbf{t}$ can be firstly determined with a objective of fully matching $G(f)$ while still respecting emission restrict $M(f)$. Based on the novel RBF framework, the frequency-domain waveform can be derived from $S(f)=$ $\sum_{i} w_{i} p\left[\left(f-f_{i}\right) / \mu_{i}\right](i=1,2, \ldots, n)$, where the vector $\mathbf{w}=\left[w_{1}\right.$, $\left.w_{2}, \ldots, w_{n}\right]^{\mathrm{T}}$ is referred to as network weight. The function $p(f)$ denotes the adopted basis function, whose position and shape may be adjusted by both $f_{i}$ and $\mu_{i}$. Then, the parameters of RBF network, including network weight $\mathbf{w}$, the position and shape of basis functions (i.e., $f_{i}$ and $\left.\mu_{i}\right)$, are modified iteratively until the error signal between the network output $S(f)$ and expected output $G$ $(f)$ reaches the minimum value. Practically, based on the error $G(f)-S(f)$, the popular gradient decent rule can be used during RBF parameters adaptation [33]. This adjusting process is shown by the solid line in Figure 4 . By introducing a promising spectrum pruning technique, furthermore, slight adjustments will be performed on partial network weigh $\mathbf{w}$ after convergence, so as to obtain satisfactory spectrum efficiency and simultaneously meet specific spectrum constraints. The process corresponding to this feedback process is depicted by the dotted line in Figure 4.

The convergence performance of mean square error (MSE), between the designed emission spectra and the target energy-efficient spectra, has been shown in Figure $5 \mathrm{a}$. It is clearly seen that, the convergence of RBFNN can be basically achieved after 50 iterations. Thus, the short switching time is practically applicable when the interference power $I(f)$ changes dynamically. Also, the adopted computationally efficient algorithm may only consume limited energy.

Notice that, when $I(f)$ is much larger than $M(f)$, the $G$ $(f)$ in specified bands should be set to 0 , which corresponds to the interference mitigation issue in the context of cognitive radios (CRs) $[17,33]$. The designed WF waveform has been illustrated in frequency domain by Figure $5 b$, in which the regulated FCC emission mask is used [13]. From the perspective of spectral matching, the designed waveform can indeed efficiently realize the optimal power allocation under arbitrary interference distribution. Thus, the optimal channel capacity can be achieved given the total emission power $P$. By optimally allocating emission power, the energy efficiency of USCS transmission can be maximized by RBFNN based WF waveform. At the same time, we may easily see that the numerically derived spectra efficiency of this RBF inspired waveform generator can even approach 97.6\%. This is much superior to the Hermit Gaussian functions-based method, whose achieved spectrum efficiency is only about $65 \%$ [34], and also significantly surpasses the second-order cone programming-based technique with a resulting efficiency of $78.8 \%$ [35]. Accordingly, from a whole system design perspective, the transmission performance can be improved by 1.7 and $0.9 \mathrm{~dB}$, respectively. Except for this practical advantage in performance, the required equivalent filter orders are also remarkable reduced, leading to a much simpler hardware implementation.

It should also be noted from Figure $5 \mathrm{~b}$ that, during the narrow notch located at $0.9-1.67 \mathrm{GHz}$ in FCC spectra mask [13], the generated waveform may surpass 


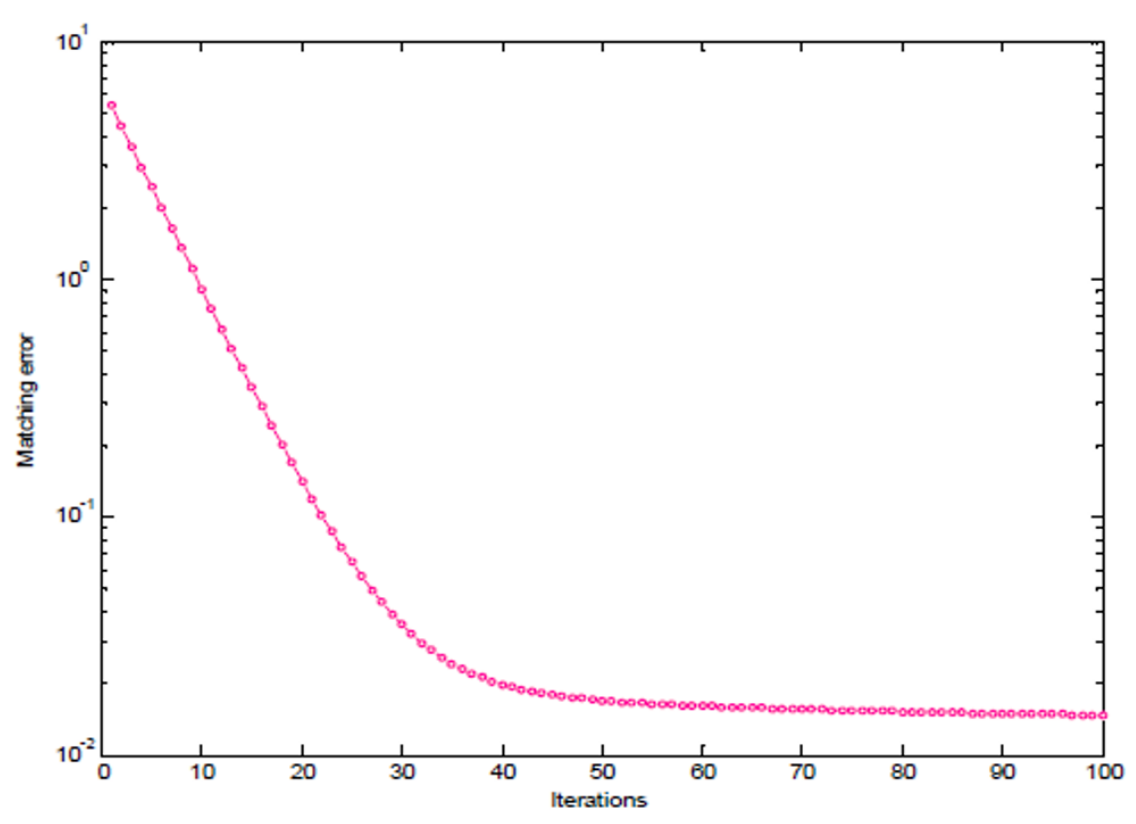

(a)

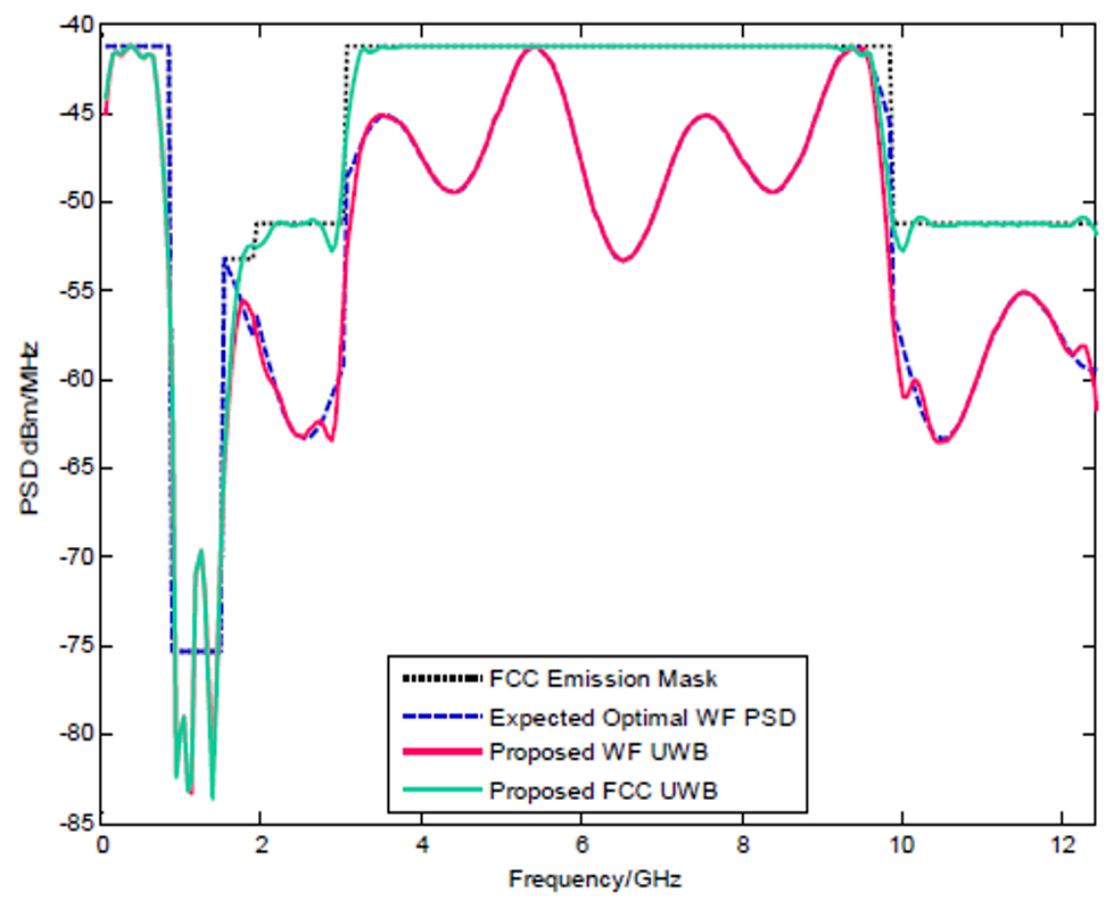

(b)

Figure 5 Performance of RBFNN-based waveform shaper. (a) MSE of RBFNN algorithm; and (b) the generated WF waveform spectrum.

emission constraint, which may arouse undesirable interference to some systems. This case can be alleviated either by using more basis functions or by introducing more complex remedy strategy, but at the expense of complicating hardware implementations. We also observed that, for the other regulated masks without such narrow spectra notches, the designed waveform may basically respect the predefined emission mask. 


\subsection{Detections in receiver \\ 3.2.1. Synchronization}

Similar to the typical UWB propagations, the high fractional bandwidth of USCS may also produce numerous resolvable multipath components in short-range applications. During recent channel measurement campaign directed at the working band ranging from 6 to $9 \mathrm{GHz}$, which seems to be the mostly overlapped band of various masks around the world, the vector network analyzer of Agilent N5242A-PNA is adopted [36]. The frequency sweeping interval between two measurement points is set to $1.5 \mathrm{MHz}$. From the extracted CIRs, it is easily observed that the received signal $r(t)$ is highly dispersive. Generally, the $85 \%$ channel energy is distributed into about 100 resolvable multipath components.

Hence, it is remaining a challenging work for accurate timing in receivers. In order to guarantee detection performance, the maximum timing error is generally about $1 \%$ of pulse duration (e.g., about 5 ps). For the most popular timing technique based on dirty-templates (TDT), integrate-and-dump operation is performed on the products of multiple symbol-long successive segments signal $[5,29]$. Various analog delays on received signal are required to estimate the practical delay $\tau_{0}$ which can maximize the integrate-and-dump output. For one thing, the analog delays can be hardly realized with sufficient precision, resulting in considerable performance degradation. Moreover, exhaustive search of practical delays ranging in $\left[0, T_{b}\right]$ is not only time-consumed but also power demanding.

$$
\tau_{0}=\underset{\tau \in\left[0, T_{b}\right)}{\arg \max } \sum_{k=1}^{K}\left(\int_{(2 k-1) T_{b}}^{2 k T_{b}} r(t+\tau) r\left(t+\tau-T_{b}\right) d t\right)^{2}
$$

In sharp contrast to the above timing technique, the precise synchronization in USCS can be basically guaranteed by one single PLL device, without any hardware difficulty as well as power-consumed search process. In practice, the timing error variance of PLL can be expressed into [28]:

$$
\varepsilon=\frac{1}{2 \alpha^{2} D} N_{0} B_{\mathrm{eq}}
$$

where $N_{0}$ is the background noise power, $B_{\text {eq }}$ is the equivalent bandwidth of PLL with a conservative value of $50 \mathrm{~Hz}$ in this USCS. $\alpha$ represents the attenuation index of free space with a typical value of 2 for single frequency [28]. Specifically, if $N_{0}$ is assumed to be $3 \times$ $10^{-17} \mathrm{~W} / \mathrm{Hz}$ (e.g., GPS frequency band) and $D$ is $1.5 \times$ $10^{-2}$, the resulting timing error variance $\varepsilon$ is about $2 \times$ $10^{-13}$ which is only $0.04 \%$ of a typical impulse duration (e.g., $0.5 \mathrm{~ns}$ ). That is, a nearly perfect timing can be basically achieved. In practice, this achieved accurate synchronization may greatly facilitate the subsequent signal detections of USCS. Compared to the existing popular timing schemes, e.g., TDT technique, the corresponding detection performance can be improved by 20 $\mathrm{dB}$ even in the absence of pilot data [29]. Specifically, when the BER drops below $10^{-3}$ and the segments number $K$ is assumed to be 4 , the required SNR (per symbol) for TDT method may even approach $37 \mathrm{~dB}$ [29]. Under a comparable BER performance, however, the required SNR (per symbol) is only about $16 \mathrm{~dB}$ in this highly synchronized system. That is, the transmit power of USCS can be further decreased by $20 \mathrm{~dB}$ given an approaching QoS. Meanwhile, by avoiding exhaustive search process in the TDT scheme, the energy consumption during timing acquisition can also be significantly reduced in receiver, with the aid of the embedded synchronized-carrier signaling. Notice that, however, the well-designed PLL with high-precision acquisition ability is expected in order to achieve the above benefits.

\subsubsection{Low-complexity detection}

Except for the aroused challenges in synchronization, the intensive multipath propagations also greatly complicate signal detection of USCS, which makes the lowpower architecture not available to realistic application $[11,12,14]$. As a low-complexity and suboptimal alternative, energy detection (ED) has drawn great interests recently in low-power scenarios [37]. In ED, the demodulation of received signal is simply to probe whether there is sufficient signal power available in specific time bins [38]. For elaboration simplicity, we may assume: (1) The synchronization has been accurately achieved; (2) the sampling rate is the Nyquist rate [28], which means each resolvable multipath is represented by one sample. Consequently, the detection problem in ED can be formulated as the following two hypotheses [38].

$$
\gamma(n)= \begin{cases}h(n)+w(n) & H_{1} \\ w(n) & H_{0}\end{cases}
$$

where $h(n)$ is the received signal, and $w(n)$ represented addition white Gaussian noise with a variance of $\sigma_{w}^{2}$.

Although with the merit of low-complexity and lowpower implementation, the detection performance of ED is hardly optimistic in practice, as no channel characteristic information can be exploited. Moreover, the performance of ED totally relies on the optimal decision threshold, which is closed related to $\sigma_{w}^{2}$. Therefore, the evitable noisy estimation uncertainty in wireless environments may further paralyze receiving performance [39].

In this section, we suggest a low-complexity noncoherent detection algorithm from an appealing pattern classification point of view. Our scheme firstly establishes a characteristic representation of multipath 
signals. Different features that fully reflect the received signal are extracted to form a feature hyperspace [40]. This high-dimensional feature space is further mapped to $2 \mathrm{D}$ plane, and signal detection is then transformed to a data clustering problem [41]. Finally, fuzzy c-means clustering algorithm is adopted to perform blind signal detection. Under low-power conditions, our scheme is robust to channel noisy uncertainty and much superior to traditional noncoherent methods (e.g., ED). Starting from the received waveform $y(n)$, our method deals with USCS detection as a two-groups data clustering by the following steps. (1) Construct the features; (2) Extract the features; (3) Combine the features; and (4) detect feature-based signals.

Features construction Given the observation consisting of $N$ discrete samples, denoted by $y(n), n=0,1,2, \ldots, N$ 1 , we firstly construct a covariance matrix $\mathbf{A}=\mathbf{y}^{T} \mathbf{y}$. Obviously, in ED the decision variable $Y_{\mathrm{ED}}$ can be also derived from the diagonal elements, i.e., $Y_{E D}=\sum \operatorname{diag}(\mathbf{A})$, where $\operatorname{diag}($.) denotes the diagonal elements. In order to enhance dissimilarity between received multipath signal (i.e., $H_{1}$ ) and channel noise (i.e., $H_{0}$ ), we further derive a symmetrical matrix by $\mathbf{B}=\mathbf{A}^{T} \mathbf{A}$. Then, we denote the principal diagonal elements of $\mathbf{B}_{N \times N}$ with $\mathbf{a}_{N \times 1}$, and the elements immediately below diagonal with $\mathbf{b}_{(N-1) \times 1}$.

$$
\begin{aligned}
& \mathbf{a}_{N \times 1}=\operatorname{diag}(\mathbf{B})=B(i, i), \quad i=0,2, \cdots, N-1 \\
& \mathbf{b}_{(N-1) \times 1}=B(i+1, i), i=1,2, \cdots, N-1
\end{aligned}
$$

Linear correlation between $\mathbf{a}$ and $\mathbf{b}^{2}$ can be then applied, which may comprehensively capture the characteristics of multipath channels $[40,41]$.

$$
\mathbf{c}_{(2 N-2) \times 1}=\mathbf{a} \otimes \mathbf{b}^{2}
$$

where $\otimes$ denotes the correlation operation. Before further proceeding, we smooth the derived sequence, $c$ $(k), k=-N+1, \ldots, 0,1, \ldots, N$, through a moving average filter, which can be realized by an equivalent matrix $\mathbf{H}$ [42]. Finally, we obtain $\mathbf{d}_{(2 N-2) \times 1}=\mathbf{H c}$. This developed representative waveform, $d(k)$, has been shown in Figure 6.

Feature extraction From Figure 6a, b, it is apparent that certain distinguished features exist in the two representative waveforms, corresponding to the channel states " $H_{1}$ " and " $H_{0}$ ", respectively. Hence, we may reasonably regard $d(k)$ as a characteristic spectrum. The quantitative features can be extracted according to the following process $[40,41]$.

First, we observe that the equivalent energy, which corresponds to the area below the waveform $d(k)$, is quite concentrated at the center of the waveform when the multipath signal has been received. Otherwise, the equivalent energy is relatively dispersed. Thus, we define the first feature as the energy concentration.

$$
F_{1}=\sum_{k=N-1-K}^{N-1+K} d^{2}(k) / \sum_{k=1}^{2 N-1} d^{2}(k)
$$

where the interested range is limited by $K$. In practice, $K$ can be determined by the imbalance property of $d(k)$. That is, $K$ can be obtained once the left value $d(N-1+$ $K)$ surpasses the right value $d(N-1-K)$ by $\zeta$. In the simplest case, we may directly set $\zeta$ to 0 .

$$
K=\min \underset{k}{\arg }\{d(N-1+k)-d(N-1-k)>\zeta\}
$$

Second, it should be noted that the slop of $d(k)$ in Figure $6 \mathrm{a}$ is much more obvious than that in Figure $6 \mathrm{~b}$ during the middle range. And consequently, the waveform variance in this range is supposed to be much distinctive. So, we define the second feature as the middle standard deviation:

$$
F_{2}=\sum_{k=N-1-K}^{N-1+K}\left(d(k)-\frac{1}{2 K-1} \sum_{k=N-1-K}^{N-1+K} d(k)\right)^{2}
$$

Third, the energy item presents remarkable imbalance during the outside range in Figure 6a, while keeping comparable in Figure 6b. Therefore, we may reasonably employ the energy imbalance as the third feature:

$$
F_{3}=\sum_{k=N-1+K}^{2 N-1} d(k)-\sum_{k=1}^{N-1-K} d(k)
$$

At last, the total received energy can also differentiate the multipath signals from the channel noise as is in ED scheme:

$$
F_{4}=\sum_{k=1}^{N} y^{2}(k)
$$

Feature combination Based on above elaborations, we have taken full advantage of USCS channel and established a quantitative feature set $\mathbf{F}=\left[\begin{array}{llll}F_{1} & F_{2} & F_{3} & F_{4}\end{array}\right]$, which is dedicated to separate two channel states, i.e., $H_{1}$ and $H_{0}$. Then, the detection of received signal can be formulated to determining a separating hyper-plane which divides the two-group pattern objects in a multidimensional features space, under the minimum classification errors [42]. Nevertheless, the solution for such a highdimensional pattern classification problem is either computationally complex or has an intolerable convergence, by consuming a mass of energy [43]. Alternatively, we may further project (or compress) feature space into the $2 \mathrm{D}$ plane by feature combinations: 


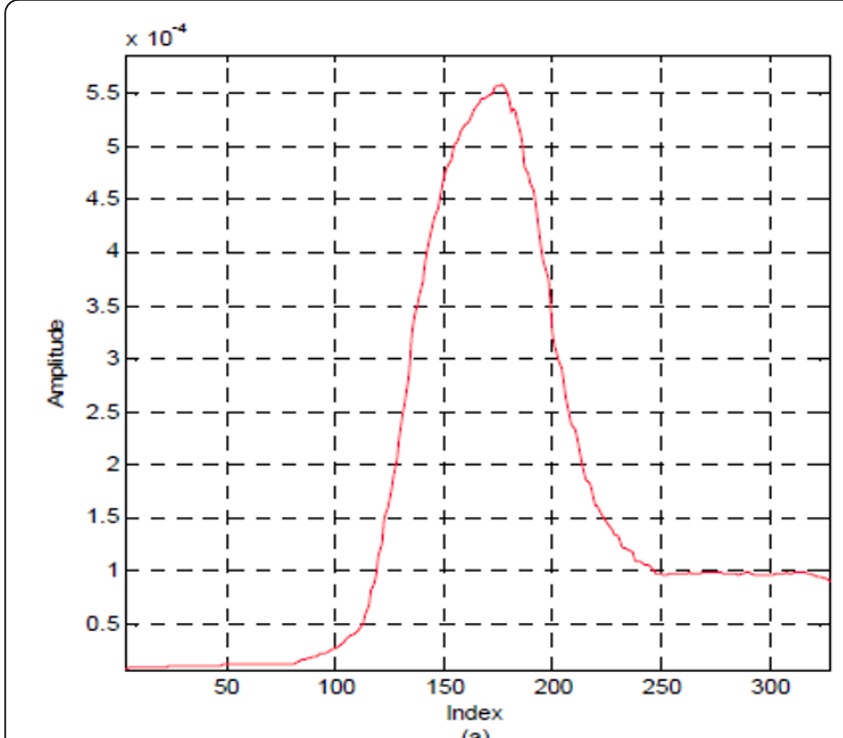

(a)

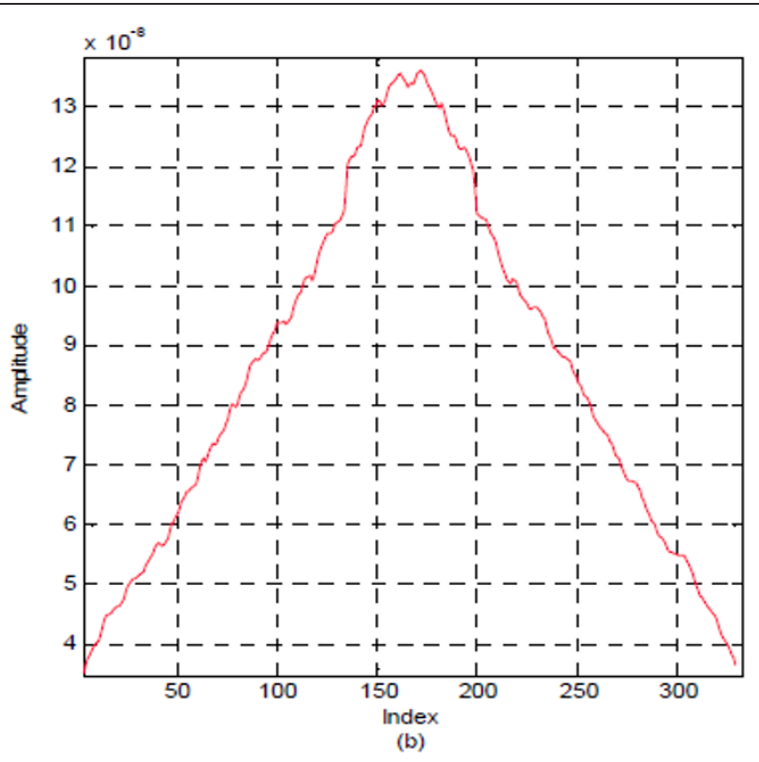

(a) Under $H_{1}$; (b) under $H_{0}$.

$$
\begin{aligned}
& v_{1}=F_{2} \\
& v_{2}=\eta_{1} F_{1}+\eta_{2} F_{4}+\eta_{3} F_{3}
\end{aligned}
$$

where the combining coefficients, i.e. $\eta_{i}(i=1,2,3)$, can be chosen according to the equal-ratio combing rule, where $\eta_{i}$ is simply proportional to mean $\left(F_{i}\right)[40,42]$.

FCM blind signal detection As the multipath signal detection is now equivalent to a data clustering problem given the $v_{1}-v_{2}$ feature plane, we may blindly divide the received data into two groups (clusters) and hence realize noncoherent signal detection. Thus, the numerous energy-consuming training data as well as the computational training algorithm can be avoided, leading to a competitive low-power USCS receiver.

Generally, clustering algorithms divide up a data set into clusters (or groups), where similar pattern objects are assigned to the same cluster whereas dissimilar pattern objects should belong to different clusters. In real applications, however, there is very often no sharp boundary between clusters, so that fuzzy clustering is often more suitable.

In fuzzy clustering, membership degrees between zero and one are used instead of crisp assignments of the data during clustering. The most prominent fuzzy clustering algorithm is the fuzzy c-means clustering [43]. In FCM, a feature vector $\left[\begin{array}{ll}v_{1} & v_{2}\end{array}\right]$ belongs to one cluster to some degree that is specified by the membership matrix $\mathbf{U}$ [19]. FCM analyzes the feature data and produces outputs: (1) the membership matrix $\mathbf{U},(2)$ the cluster centers $\{$ center_i, $i=1,2\}$. Four steps in FCM are depicted below.
Step 1: Initialize the membership matrix $U$ with random values between 0 and 1 . The size of $\mathbf{U}$ is $Q \times P$, where $Q$ is the number of clusters, and $P$ is the number of input feature vectors. The element of membership matrix $\mathbf{U}$, i.e., $u_{i j}$, is the probability that the $j$ th feature vector belongs to the $i$ th cluster $(1 \leq i \leq Q$ and $0 \leq j \leq$ $P$ - 1). It is noted that, for a given feature vector, the summation of degrees of belongness equals unity. Therefore, elements of $\mathbf{U}$ must satisfy the constraint below:

$$
\sum_{i=1}^{Q} u_{i j}=1, \quad 0 \leq j \leq P-1
$$

Step 2: Calculate fuzzy cluster centers for all $Q$ possible clusters according to

$$
\text { Center_ } i=\sum_{j=1}^{P-1} v_{j} u_{i j} / \sum_{j=1}^{P-1} u_{i j}, 0 \leq i \leq Q
$$

where $v_{j}$ is the feature vector derived from spectrum harmonics coefficients defined in (18). Here, $Q=2$ for our analysis.

Step 3: Compute the cost function defined below:

$$
J(U, \text { center_ } 1, \text { center_2 })=\sum_{i=1}^{Q} \sum_{j=1}^{P-1} u_{i j}{ }^{m} D_{i j}^{2}
$$

and

$$
D_{i j}=\| \text { center }_{-} i-v_{j} \|
$$

where (22) calculates the Euclidean distance between the $i$ th cluster center and the $j$ th feature vector. In (21), the weighting exponent is assumed to be $m=2[19,43]$. 
The iteration criterion requires the cost function $J$ to be as small as possible. The iteration terminates if improvement of $J$ over previous iteration is below a pre-specified threshold. Otherwise, the algorithm proceeds with next step to update the membership matrix $\mathbf{U}$.

Step 4: Compute a new membership matrix $\mathrm{U}$ whose element $u_{i j}$ is adjusted by

$$
u_{i j}=\sum_{k=1}^{Q}\left(D_{i j} / D_{k j}\right)^{2 / 1}-m 1 \leq i \leq Q, 0 \leq j \leq P-1
$$

According to the above equation, $u_{i j}$ is inversely proportional to the squared distance from $v_{j}$ to the cluster center center $i$.

The above iteration process, from Steps 2 to 4, continues on the input patterns, until either the predefined maximum iteration has been reached or the objective function $J$ has been sufficiently converged.

We first show the convergence performance of the objective function $J$ of FCM in Figure 7a. In this experiment, we set channel $E_{b} / N_{0}$ to $14 \mathrm{~dB}$, the maximum iteration is 150 and the stop criterion on the relative minimum improvement is $10^{-5}$. It is clearly observed that this clustering algorithm can reach its convergence after ten iterations. Therefore, the detection algorithm is relatively computationally efficient, which is suitable to real-time applications. The derived 2D feature plane is then obtained through the proposed algorithm above, as illustrated in Figure 7b. We note from the simulation result that our scheme can essentially cluster the received USCS signals into the right groups in a totally blind fashion, without any assistance of energy-inefficient training patterns (e.g., pilot sequence). Based on numerical derivations, the detection bit error rate (BER) of the FCM-based noncoherent algorithm is about $1.3 \times$ $10^{-5}$. By comparison, even the noise variance has been accurately estimated, the BER still remains about $4.7 \times$ $10^{-3}$ for noncoherent ED. So, within a low-power framework the proposed noncoherent scheme may considerably enhance receiving performance.

Based on the Monte Carlo experiment, the detection BER curves obtained from numerical derivations have also been plotted in Figure 8. In the simulations, the modified S-V channel modeling regulated by the IEEE 802.15.3a task group is adopted [10,11], with the resolvable multipath components of $N=150$. By exploring the strong carrier singling, the accurate timing is assumed by using PLL (i.e., the timing error variance is $0.04 \%$ of the impulse duration $T_{c}$ ). It should also be noted that, for the presented noncoherent scheme, highorder modulation schemes with high-data rate may become valid as the phase information has been totally lost. Thus, the simple and popular PPM is adopted. When the channel noise variance $\sigma_{w}^{2}$ is estimated accurately, our pattern classification-based noncoherent algorithm can obtain a gain about $1.4 \mathrm{~dB}$ compared with classical ED. Furthermore, when channel noise uncertainty is taken into consideration, a receiving gain about 4-5 $\mathrm{dB}$ can be achieved when employing our presented noncoherent detector, given no channel noise information is used in our blind algorithm. Hence, our method is superior to ED and also robust to practical noise uncertainty. Although it is relatively cumbersome to evaluate the reduction of energy consumption in this noncoherent receiver, with a computation complexity order of $O\left(N^{L}\right)(2<L<3)$ and a simplified hardware structure, our suggested method is much more competitive compared to the exponential complexity order of classical CIR estimators and the power-demanding multiple correlation fingers (i.e., Rake structure).

\section{Energy-efficient green communications}

In this section, we will give a budgetary analysis for energy efficiency of both USCS and traditional approaches (i.e., BPSK and UWB-IR). First, we investigate the traditional intermittent UWB-IR technique. Given that the information-bearing waveforms with a typical short duration of $0.5 \mathrm{~ns}$ and a LDC value of $1 \%$, the occupying bandwidth of UWB is about $2 \mathrm{GHz}$. Nevertheless, the achieved symbol rate is only about 20 MHz. Consequently, as indicated by Win and Scholtz [6], this impulse modulation based underlay transmission belongs to a special SS system, with a spreading factor $\mu$ of 100 (i.e., $20 \mathrm{~dB}$ ), which is strikingly different from the pseudorandom code based SS mechanisms (e. g., CDMA). For UWB-IR, this introduced SS gain allows UWB transceiver to operate at a rather low emission power. Usually, the expected operational power spectrum density may keep below $-41.3 \mathrm{dBm} / \mathrm{MHz}$ [5], while transmission performance of UWB devices can be basically guaranteed due to this spreading gain (e.g., $20 \mathrm{~dB}$ ). Practically, such a gain can be achieved by using a highly synchronized gating operation on received signals, which considerably excludes noise outside information durations. Hence, the high energy efficiency of single UWB link is essentially attributed to the reduced spectra efficiency and the resulting tremendous spreading gain. Fortunately, TH-PPM scheme can be employed to provide multiple accesses and hence improve the capacity of UWB network.

\subsection{UWB versus BPSK}

For conventional BPSK systems, the information bit is modulated into carrier waveform that lasts for the whole symbol duration. Thereby, the signal bandwidth is exactly equivalent to data rate, that is, the spreading gain $\mu$ is 1 (i.e., $0 \mathrm{~dB}$ ). Given the total emission bit power $E_{b}$ and the channel noise power $N_{0}$, the receiver 


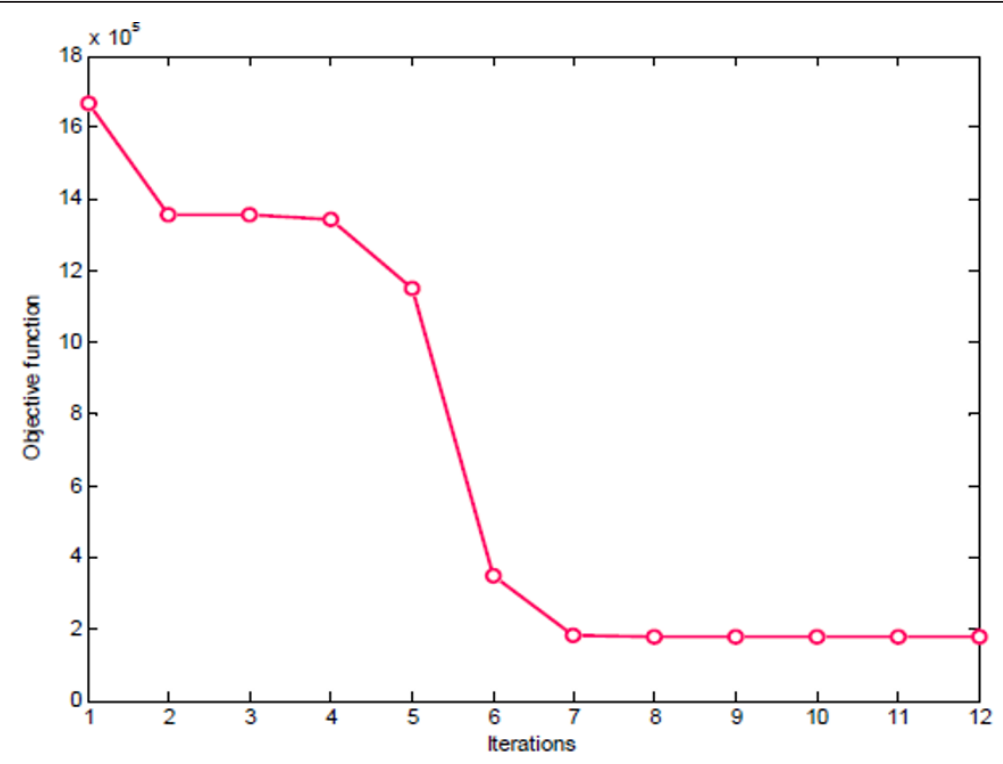

(a)

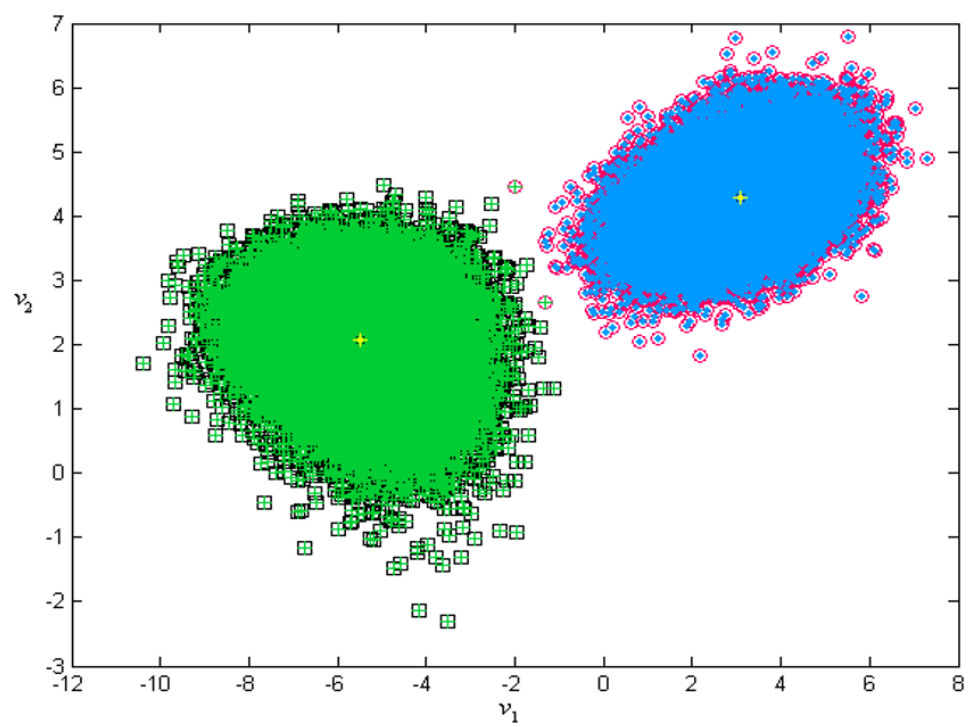

(b)

Figure 7 Performance of FCM method. (a) FCM cost function versus iterations; (b) Pattern representations of received signals. In (b), notice that the patterns labeled by squares and circles represent the real transmitted bit " 0 " and "1", while the patterns labeled by " + " and "." denote the clustering result.

SNR of BPSK is $E_{b} / N_{0}[28]$. On the other hand, for UWB-IR systems, the emission power in the intermittent mode is only about $0.01 \times E_{b}$, and the original SNR is reduced to $0.01 \times E_{b} / N_{0}$. While the emission power in UWB transceiver is only $1 \%$ of BPSK system under the comparable detection performance, by taking fully advantages of spreading gain $\mu^{\prime}$ which is rightly $20 \mathrm{~dB}$, the detection performance is actually equal to BPSK, i.e. $\mu^{\prime} \times 0.01 \times E_{b} / N_{0}$ [28]. Therefore, the energy efficiency of UWB is rather obvious compared to the BPSK scheme. As an expense of this transmission advantage, nevertheless, the detection performance of UWB receivers is remarkably sensitive to synchronization deviation [5], while the precise timing always increases UWB receiver energy consumptions greatly. For example, a $1 \%$ synchronization deviation is about $5 \times 10^{-10} \mathrm{~s}$ for BPSK, 


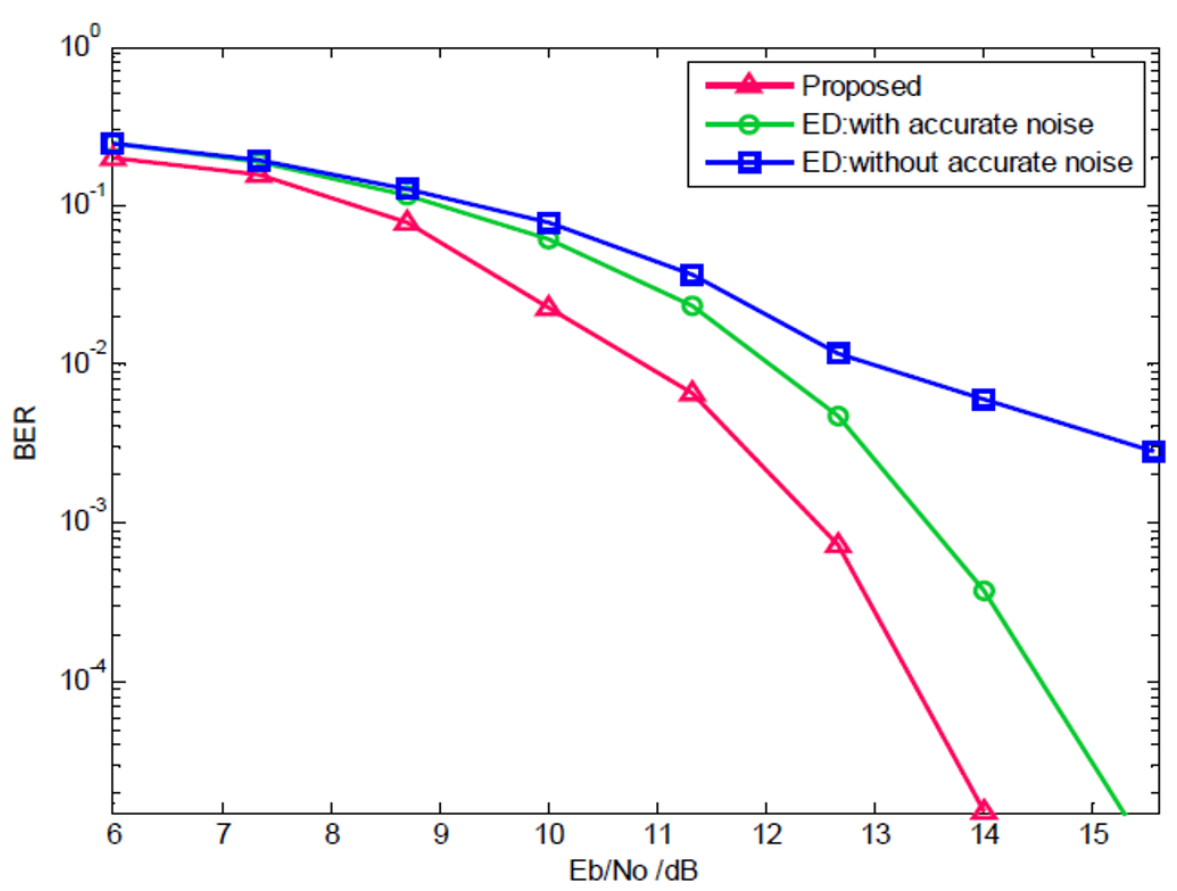

Figure 8 BER performance of the noncoherent USCS receiver.

while is even about $5 \times 10^{-12} \mathrm{~s}$ for UWB impulse system.

\subsection{USCS versus UWB}

Compared to UWB-IR system having a receiving SNR of $E_{b} / N_{0}$, e.g., still given the total emission bit power $E_{b}$ and the channel noise power $N_{0}$, a comparable receiver SNR may increase the transceiver power even to $100 \times$ $E_{b}$ for the suggested carrier-synchronized USCS. On the other hand, this strong carrier-synchronized components in USCS can be extracted by PLL to facilitate the low-complexity and precise synchronization/timing. Moreover, this precise timing information may produce an additional SNR saving of $20 \mathrm{~dB}$ (or even much) in USCS receiver. Therefore, given an expected QoS and the same channel conditions, the emission power of USCS can be further reduced to $E_{b}$ (i.e., $100 \times E_{b} / 10^{20 /}$ $\left.{ }^{10}\right)$, which is basically equivalent to UWB transceiver power. More importantly, the power-consumed synchronization-aiding data in transmitter and the complicated synchronization operations in receiver can be avoided, resulting in a remarkable energy saving for USCS. For example, other than the analog processes (i.e., low-noise amplifier and ADC), the digital synchronization operation may even accounts for $30-40 \%$ energy consumption of receivers [37].

Consequently, from a systematic perspective, the energy efficiency of USCS is much superior to both BPSK and UWB-IR even in continuous carrier- synchronized mode, which essentially provides one competitive approach to green communications. If reconfigurable emission in transceiver is considered, furthermore, the USCS energy efficiency may become much more conspicuous with the significantly reduced energy consumption in transmitter. Assuming an operational time ratio between the two hybrid modes (i.e., continuous emission and intermittent emission) is $10 \%$, then a schematic ultra-low-power communication architecture with energy consumption far less than $400 \mu \mathrm{W}$, i.e., $1 \mathrm{~mW} \times[10 \% / 2+(1-30 \%) / 2]$, can be basically promised without taking the potential advantages of WF waveform design and low-complexity detection. Notice that, in our above rough evaluation, the first term (i.e., $10 \%$ ) accounts for the combined benefits from both the precise synchronization and reconfigurable emission, while the second (i.e., 30\%) represents the energy saving introduced by the simplified timing process.

\subsection{Other application of USCS}

The significance of large-scale WSNs has been emphasized by the emerging internet of things. Usually, the great numbers of sensor nodes are battery-powered due to the considerations of low-cost and ease of deployment. In the super large-scale networks, unfortunately, energy management issues may tend to beyond control in practice. For a sensor network containing tens of thousands of distributed nodes, the network lifetime may even be shortened to several hours because of the 
tremendous and frequent information exchange among sensors, which quickly consumes most of battery energy. As another potential application, the presented USCS can be properly applied to provide a feasible remote powering scheme for the distributed large-scale sensor nodes. As indicated, the single-frequency component can be conveniently recovered by a PLL. Other than providing precise timing for sensor nodes, the receiver can also reserve this carrier energy for subsequent operations by resorting to wireless charging technique. Hence, in large-scale WSNs, the lifetime of low-cost and battery-powered sensors can be significantly prolonged. In fact, the device prototype has recently been developed by CEA-Leti and Nokia Researchers [18], which is much similar to our presented USCS philosophy that integrates UWB together with the ultra-narrow band ultrahigh-frequency (UHF) signaling.

\section{Discussions and conclusions}

In this article, we suggest a promising transmission scheme for energy-efficient green communications. The presented USCS is mainly inspired by the underlay communication and LDC mechanic, in which information data is modulated into baseband waveforms with an extremely short duration. Simultaneously, a SCM technique is constructively integrated into emission signals. The resulting carrier-synchronized signal can greatly facilitate the precise timing acquisition and enhance receiving performance of this synchronization-sensitive system. Also, the suggested hybrid emission strategy allows transceiver operating at a low-power radiation condition. Relying on RBFNN, the optimal water-filling emission waveform is designed to maximize the transmission energy efficiency in the presence of external interference. In order to minimize energy consumption in receiver, a low-complexity noncoherent detection scheme is further proposed based on a blind FCM technique. The comparative performance evaluation with either traditional BPSK or UWB-IR indicates that the presented systematic USCS can be realized within an ultra-low-power framework (e.g., less than $400 \mu \mathrm{W}$ ), which shows great potential in future short-range green communication networks. This framework also suggests a feasible energy management solution to battery-powered sensor networks, greatly facilitating widespread applications of the large-scale distributed WSNs.

\footnotetext{
Acknowledgements

This study was supported by the National Science \& Technology Major Projects (2009ZX03006-009), NSFC (60772021) the BUPT Excellent Ph.D. Students Foundation (CX201122) and the SCIE Excellent Graduate Students Foundation. This study also received support from the MIKE (The Ministry of Knowledge Economy), Korea, under the ITRC (Information Technology Research Center) support program supervised by the NIPA (National IT Industry Promotion Agency) (NIPA-2011- C1090-1111-0007).
}

Competing interests

The authors declare that they have no competing interests.

Received: 29 August 2011 Accepted: 15 February 2012

Published: 15 February 2012

\section{References}

1. MR Bloomberg, Inventory of New York City Greenhouse gas emissions. Technical Report (April 2007)

2. R Bunden, White paper green ICT. Technical Report, T-Systems Enterprise Service $\mathrm{GmbH}$ (2008)

3. R Hodges, W White, Go green in ICT.2008 (2008) Technical Report, Green Tech News

4. P Cheolhee, TS Rappaport, Short-range wireless communications for nextgeneration networks: UWB, $60 \mathrm{GHz}$ Millimeter-Wave WPAN, and ZigBee. IEEE Wirel Commun. 14(4), 70-78 (2007)

5. LQ Yang, GB Giannakis, Ultra-wideband communications: an idea whose time has come. IEEE Signal Process Mag. 21(6), 26-54 (2004). doi:10.1109/ MSP.2004.1359140

6. MZ Win, RA Scholtz, Ultra-wide bandwidth time hopping spread spectrum impulse radio for wireless multiple-access communications. IEEE Trans Commun. 48(4), 679-689 (2000). doi:10.1109/26.843135

7. MZ Win, RA Scholtz, Impulse radio: how it works. IEEE Commun Lett. 2(2), 36-38 (1998). doi:10.1109/4234.660796

8. S Roy, JR Foerster, VS Omayazulu, DG Leeper, Ultra-wideband radio design: the promise of high-speed, short-range wireless connectivity. Proc IEEE. 92(2), 295-311 (2004). doi:10.1109/JPROC.2003.821910

9. M Chen, S Gonzalez, A Vasilakos, HS Cao, VCM Leung, Body area networks: a survey. Mobile Netw Appl (2010). doi:10.1007/s11036-010-0260-8

10. MZ Win, RA Scholtz, Characterization of ultra-wide bandwidth wireless indoor channels: a communication-theoretic view. IEEE J Sel Areas Commun. 20(9), 1613-1627 (2002). doi:10.1109/JSAC.2002.805031

11. A Molisch, Ultrawideband propagation channels-theory, measurement, and modeling. IEEE Trans Veh Technol. 54(5), 1528-1545 (2005). doi:10.1109/ TVT.2005.856194

12. WM Lovelace, JK Townsend, The effects of timing jitter and tracking on the performance of impulse radio. IEEE J Sel Areas Commun. 20(9), 1646-1651 (2002). doi:10.1109/JSAC.2002.805058

13. (FCC) Federal Communications Commission, Revision of part 15 of the commission's rules regarding ultra-wideband transmission systems. First Report and Order Released: (April 2002)

14. A Rajeswaran, VS Somayazulu, J Foerster, Rake performance for a pulse based UWB system in a realistic UWB indoor channel. in IEEE ICC'03, Anchorage, Alaska, USA, 4, 2879-2883 (May, 2003)

15. S Franz, U Mitra, Generalized UWB transmitted reference systems. IEEE J Sel Areas Commun. 24(4), 780-786 (2006)

16. B Li, Z Zhou, WX Zou, RPPK modulation with high data rates. Sci China Inf Sci. 53(2), 344-354 (2010). doi:10.1007/s11432-010-0012-1

17. Z Zhou, B Li, QJ Song, Open spectrum and UWB research in China: spectrum reform and radio revolution. in Proc IEEE Int Conf Ultra-Wideband (ICUWB 2010), Nanjing, China, 2, 1-5 (September, 2010)

18. M Pelissier, B Gomez, G Masson, S Dia, M Gary, J Jantunen, J rponen, J Varteva, A $112 \mathrm{Mb} / \mathrm{s}$ full duplex remotely-powered impulse-UWB RFID transceiver for wireless NV-memory applications. in Proc IEEE Symposium on VLSI Circuits (VLSIC 2010), Honolulu, Hawaii, USA, 46, 25-26 (June, 2010)

19. JC Bezdek, Pattern Recognition with Fuzzy Objective Function Algorithms. Plenum Press, New York, 1981)

20. HG Schantz, K Siwiak, T Janik, Proof-of-concept C-UWB data link. in Proc of 2008 IEEE Sarnoff Symposium, Princeton, NJ, 1, 1-5 (April, 2008)

21. JY Hu, YL Zhu, S Wang, H Wu, Energy efficient, reconfigurable, distributed pulse generation and detection in UWB impulse radios. in Proc of IEEE International Conference on Ultra-Wideband (ICUWB 2009), Vancouver, Canada, 1, 773-777 (September, 2009)

22. MZ Win, A unified spectral analysis of the generalized time-hopping spread-spectrum signals in the presence of timing jitters. IEEE J Sel Areas Commun. 20, 1664-1676 (2002). doi:10.1109/JSAC.2002.805030

23. HR Walker, VPSK and VMSK modulation transmit digital audio and video at 15 bit/s/Hz. IEEE Trans Broadcast. 43, 96-103 (1997). doi:10.1109/11.566830

24. LN Wu, M Feng, On BER performance of EBPSK-MODEM in AWGN channel. Sensors. 10(4), 3824-3834 (2010). doi:10.3390/s100403824 
25. XY Chen, QD Yao, XC Liu, The VMSK modulation delusion. J Electron Inf Technol. 25, 1558-1563 (2003)

26. Z Baranski, AM Haimovich, Performance analysis of VPSK digital modulation. Wirel Person Commun. 19(2), 139-145 (2001). doi:10.1023/A:1011910120946

27. KH Sayhood, W Lenan, Raise bandwidth efficiency with sine-wavemodulation VMSK. Microwave RF. 40(4), 79-84 (2001)

28. J Proakis, Digital Communications, 4th edn. McGraw-Hill, New York, (2001)

29. L Yang, GB Giannakis, Timing UWB signals using dirty templates. IEEE Trans Commu. 53(11), 1952-1963 (2005). doi:10.1109/TCOMM.2005.858663

30. F Troesch, C Steiner, T Zasowski, T Burger, A Wittneben, Hardware aware optimization of an ultra low power UWB communication system. in Proc IEEE Int Conf Ultra-Wideband (ICUWB 2007), Marina Mandarin, Singapore, 1, 174-179 (September, 2007)

31. HW Yang, A road to future broadband wireless access: MIMO-OFDM-based air interface. IEEE Commun Mag. 43, 53-60 (2005)

32. WX Zou, B Li, Z Zhou, Energy efficient water filling ultra wideband waveform shaping based on radius basis function neural networks. vol. 6221/2010, (Springer Lecture Notes in Computer Science (LNCS), 2010) pp. 299-306

33. B Li, Z Zhou, WX Zou, A novel adaptive spectrum forming filter: application in the cognitive Ultra-wideband. Sci China Inf Sci. 53, 2584-2599 (2010). doi:10.1007/s11432-010-4106-6

34. RM Yang, Hermite Gaussian orthogonal functions, orthogonal basis: their applications in ultra wideband and ultra narrowband cognitive radio. Ph.D. Dissertation, Beijing University of Posts and Telecommunications, (June, 2007)

35. I Dotlic, R Kohno, Design of the family of orthogonal and spectrally efficient UWB waveforms. IEEE J Sel Topic Signal Process. 1, 21-30 (2007)

36. B Li, Z Zhou, DJ Li, SJ Zhai, Efficient cluster identification for measured ultra-wideband channel impulse response in vehicle cabin. Prog Electromagnet Res (PIER). 117, 121-147 (2011)

37. K Witrisal, G Leus, G Janssen, M Pausini, F Troesch, T Zasowski, J Romme, Noncoherent ultra-wideband systems. IEEE Signal Process Mag. 26(4), 48-66 (2009)

38. A D'Amico, U Mengali, E Arias-de Reyna, Energy-detection UWB receivers with multiple energy measurements. IEEE Trans Wirel Commun. 6(7), 2652-2659 (2007)

39. R Tandra, A Sahai, Fundamental limits on detection in low SNR under noise uncertainty. in Proc of WirelessCom 2005, Maui, HI, 1, 464-469 (June, 2005)

40. B Li, Z Zhou, DJ Li, WX Zou, A novel Parzen probabilistic neural network based noncoherent detection algorithm for distributed ultra-wideband sensors. J Netw Comput Appl. 34, 1894-1902 (2011). doi:10.1016/j. jnca.2010.12.015

41. B Li, Z Zhou, WX Zou, WX Gao, Particle Swarm Optimization Based Noncoherent Detector for Ultra-wideband Radio in Intensive Multipath Environments. EURASIP J Adv Signal Process. 2011, 1-14 (2011). paper ID: 341836

42. B Li, Z Zhou, WX Zou, SB Wang, Fuzzy C-means clustering based robust and blind noncoherent receivers for underwater sensor networks. in Proc of the 5th international conference on wireless algorithms, system and applications (WASA 2010), Beijing China, 6221/2010, 314-321, (2010)

43. OD Richard, EH Peter, GS Dvaid, Pattern Classification, 2nd edn. (John Wiley \& Sons, Inc., New York, 2001)

doi:10.1186/1687-1499-2012-47

Cite this article as: Li et al:: Ultra-spectra communication system (USCS): a promising way to energy-efficient green communication networks. EURASIP Journal on Wireless Communications and Networking 2012 2012:47.

\section{Submit your manuscript to a SpringerOpen ${ }^{\mathcal{O}}$ journal and benefit from:}

- Convenient online submission

- Rigorous peer review

- Immediate publication on acceptance

- Open access: articles freely available online

- High visibility within the field

- Retaining the copyright to your article

Submit your next manuscript at $\gg$ springeropen.com 